\title{
Urogynecology digest
}

\author{
Presented by: Alex Mowat
}

\section{Vaginal estrogen: a dual-edged sword in postoperative healing of the vaginal wall}

Ripperda CM, Maldonado PA, Acevedo JF, Keller PW, Akgul Y, Shelton JM, Word RA. Menopause. 2017 Jul;24(7):838849. doi: 10.1097/GME.0000000000000840

This randomized controlled trial was aimed at testing the hypothesis that postoperative administration of local estrogen, direct mesenchymal stem cells (MSCs) or both improves healing of the vaginal mucosa after reconstructive surgery, and may therefore improve success rates of pelvic reconstructive surgery. The same group has previously established that preoperative estrogen cream improves wound healing in ovariectomized guinea pigs [1] and that preoperative topical estrogen increases vaginal wall collagen content in postmenopausal women undergoing reconstructive pelvic surgery [2].

The trial was carried out on 170 rats that had had their ovaries removed and then underwent surgical incision of their posterior vaginal wall. There were four arms to which the rats were randomized immediately postoperatively: placebo $(n=41)$, estrogen cream $(n=47)$, direct injection of MSCs $(n=39)$, or both estrogen cream and MSCs $(n=43)$.

The trial showed that MSCs did not survive once they were injected into the vagina and so had no effect on healing. The effects of postoperative local estrogen on healing of the injured vagina were divergent; there was a positive effect on the mucosal layer but a negative effect on the early healing phase in the stromal layer. The effects on the mucosa included increased epithelial thickness over the wound, increased expression of genes related to epithelial barrier function, and increased protease inhibitors that may protect the matrix from degradation during healing. The negative effects on the stroma included decreased collagen content and decreased expression of genes for matrix components.

The authors conclude that the negative effects on the stroma outweigh the positive effects on the mucosa and, therefore, that the administration of local estrogen cream in the immediate postoperative period is not helpful for wound healing.

\section{References}

1. Balgobin S, Montoya TI, Shi H, et al. Estrogen alters remodeling of the vaginal wall after surgical injury in guinea pigs. Biol Reprod. 2013;89:138.

2. Rahn DD, Good MM, Roshanravan SM, et al. Effects of preoperative local estrogen in postmenopausal women with prolapse: a randomized trial. J Clin Endocrinol Metab. 2014;99:3728-36.

Alex Mowat

zanhmowat@gmail.com

1 Royal Brisbane Hospital, Brisbane, Australia 